LA-UR: $00-3058$

Title: Final Neutrino Oscillation Results from LSND

Author(s):

W. C. Louis (representing the LSND Collaboration)

Submitted to:

Second Tropical Workshop on

Particle Physics and Cosmology

San Juan, Puerto Rico

May $1-5,2000$

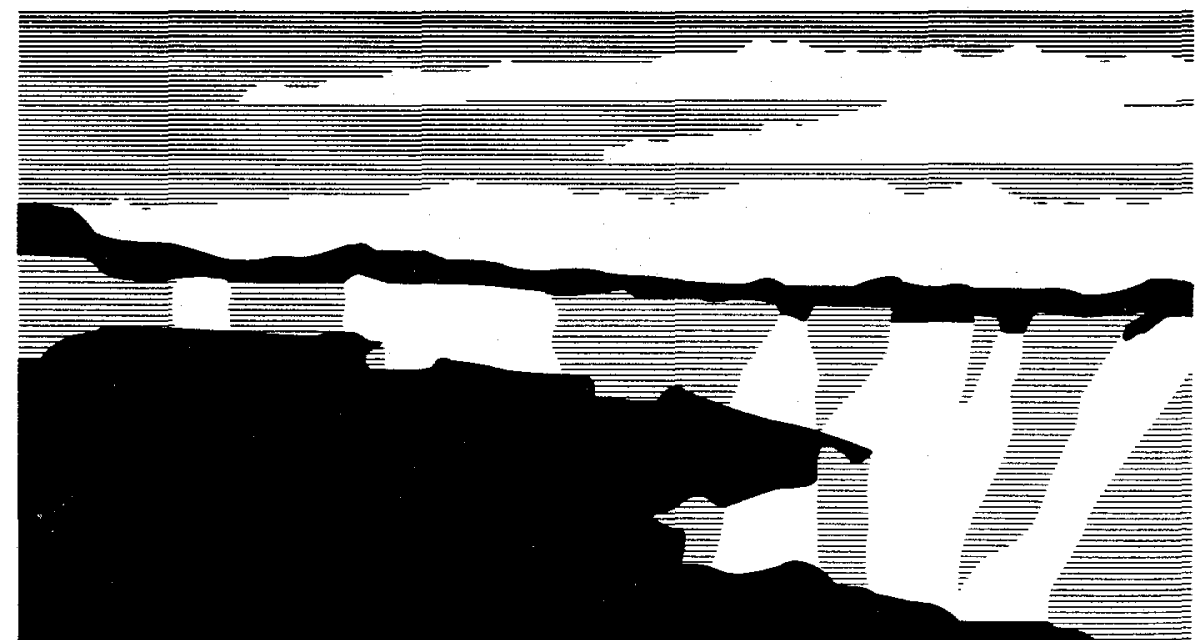

NATIONAL LABORATORY

Los Alamos National Laboratory, an affirmative action/equal opportunity emplofyer, is operated by the University of California for the U.S. Department of Energy under contract W-7405-ENG-36. By acceptance of this article, the publisher recognizes that the U.S. Government retains a nonexclusive, royalty-free license to publish or reproduce the published form of this contribution, or to allow others to do so, for U.S. Government purposes. The Los Alamos National Laboratory requests that the publisher identify this article as work performed under the auspices of the U.S. Department of Energy. 


\section{DISCLAIMER}

This report was prepared as an account of work sponsored by an agency of the United States Government. Neither the United States Government nor any agency thereof, nor any of their employees, make any warranty, express or implied, or assumes any legal liability or responsibility for the accuracy, completeness, or usefulness of any information, apparatus, product, or process disclosed, or represents that its use would not infringe privately owned rights. Reference herein to any specific commercial product, process, or service by trade name, trademark, manufacturer, or otherwise does not necessarily constitute or imply its endorsement, recommendation, or favoring by the United States Government or any agency thereof. The views and opinions of authors expressed herein do not necessarily state or reflect those of the United States Government or any agency thereof. 


\section{DISCLAIMER}

Portions of this document may be illegible in electronic image products. Images are produced from the best available original document. 


\title{
FINAL NEUTRINO OSCILLATION RESULTS FROM LSND
}

\author{
W. C. LOUIS, representing the LSND Collaboration ${ }^{1}$ \\ Physics Division, Los Alamos National Laboratory, \\ Los Alamos, NM 87545, USA \\ E-mail: louis@lanl.gov
}

\begin{abstract}
The LSND experiment at Los Alamos has conducted searches for $\bar{\nu}_{\mu} \rightarrow \bar{\nu}_{e}$ oscillations using $\bar{\nu}_{\mu}$ from $\mu^{+}$decay at rest and for $\nu_{\mu} \rightarrow \nu_{e}$ oscillations using $\nu_{\mu}$ from $\pi^{+}$decay in flight. For the $\bar{\nu}_{\mu} \rightarrow \bar{\nu}_{e}$ search, a total excess of 83.3 $\pm 21.2 \pm 12.0$ events is observed with $e^{+}$energy between 20 and $60 \mathrm{MeV}$, while for the $\nu_{\mu} \rightarrow \nu_{e}$ search, a total excess of $8.3 \pm 5.5 \pm 4.0$ events is observed with $e^{-}$energy between 60 and $200 \mathrm{MeV}$. If attributed to neutrino oscillations, the most favored allowed region from a fit to the entire data sample is a band from 0.2 to $2.0 \mathrm{eV}^{2}$. This result implies that at least one neutrino has a mass greater than $0.4 \mathrm{eV} / \mathrm{c}^{2}$ and that neutrinos contribute more than $1 \%$ to the mass of the universe.
\end{abstract}

\section{INTRODUCTION}

The LSND experiment collected data for six years, from 1993 to 1998, during which time the LAMPF/LANSCE accelerator operated for 17 months of calendar time and delivered $28,898 \mathrm{C}(\sim 0.3 \mathrm{~g})$ of protons on target. Using partial data samples, evidence for neutrino oscillations has been published previously for both $\bar{\nu}_{\mu} \rightarrow \bar{\nu}_{e}[2,3]$ and $\nu_{\mu} \rightarrow \nu_{e}[4]$ oscillations. In this report we present the final LSND oscillation results that include the entire 1993-1998 data sample, that combines the two oscillation searches in a global analysis, and that makes use of a new event reconstruction that has greatly improved the event spatial and angular resolutions. An excess of events consistent with neutrino oscillations is observed and implies that at least one neutrino has a mass greater than $0.4 \mathrm{eV} / \mathrm{c}^{2}$ and that neutrinos contribute more than $1 \%$ to the mass of the universe.

The old event position reconstruction was hampered due to the charge response of the 8" phototubes used in LSND (Hamamatsu R1408). For these phototubes, the single photoelectron distribution is essentially a broad Gaussian peak followed by an exponential charge tail that extends to arbitrarily high values. As the position and angle fits weight the hit phototubes by their charge, this charge tail has the effect of smearing the reconstructed event positions and angles. (It also has the effect of smearing the energy resolution. At $50 \mathrm{MeV}$ the electron energy resolution is $\sim 7 \%$, 
much worse than the $\sim 3 \%$ resolution that would be expected from photon statistics alone.) To ameliorate this effect, a new reconstruction was developed that weights the hit phototubes by their expected charge and not by their actual charge. This has resulted in an improvement in the correlated positions for muon decay events. The mean reconstructed distance between the muon and decay electron has improved from $22 \mathrm{~cm}$ with the old reconstruction to $14 \mathrm{~cm}$ with the new reconstruction. For $2.2 \mathrm{MeV} \gamma$ from neutron capture, the most likely distance has improved from 74 $\mathrm{cm}$ to $55 \mathrm{~cm}$.

\section{DETECTOR}

The Liquid Scintillator Neutrino Detector (LSND) experiment at Los Alamos [5] was designed to search with high sensitivity for $\bar{\nu}_{\mu} \rightarrow \bar{\nu}_{e}$ oscillations from $\mu^{+}$decay at rest. The LANSCE accelerator is an intense source of low energy neutrinos due to its $1 \mathrm{~mA}$ proton intensity and $800 \mathrm{MeV}$ energy. For the 1993-1995 running period the beam stop consisted of a $30-\mathrm{cm}$ long water target $(20-\mathrm{cm}$ in 1993) followed by a water-cooled $\mathrm{Cu}$ Beam dump, while for the 1996-1998 running period the beam stop was reconfigured with the water target replaced by a close-packed high-z target for testing tritium production. The muon decay-at-rest neutrino flux with this new configuration is only $2 / 3$ of the neutrino flux with the old beam stop; however, the pion decay-in-flight neutrino flux has been reduced to $1 / 2$ of the original flux, so that the 1996-1998 data serve as a systematic check. The neutrino source is well understood because almost all neutrinos arise from $\pi^{+}$or $\mu^{+}$decay; $\pi^{-}$and $\mu^{-}$ are readily captured in the $\mathrm{Fe}$ of the shielding and $\mathrm{Cu}$ of the beam stop. [6] The production of kaons and heavier mesons is negligible at these energies. The $\bar{\nu}_{e}$ rate is calculated to be only $4 \times 10^{-4}$ relative to $\bar{\nu}_{\mu}$ in the $36<E_{\nu}<52.8 \mathrm{MeV}$ energy range, so that the observation of a significant $\bar{\nu}_{e}$ rate would be evidence for $\bar{\nu}_{\mu} \rightarrow \bar{\nu}_{e}$ oscillations.

The LSND detector consists of an approximately cylindrical tank $8.3 \mathrm{~m}$ long by $5.7 \mathrm{~m}$ in diameter. The center of the detector is $30 \mathrm{~m}$ from the neutrino source. On the inside surface of the tank 1220 8-inch Hamamatsu phototubes provide $25 \%$ photocathode coverage. The tank is filled with 167 metric tons of liquid scintillator consisting of mineral oil and $0.031 \mathrm{~g} / \mathrm{l}$ of b-PBD. This low scintillator concentration allows the detection of both Cerenkov light and scintillation light and yields a relatively long attenuation length of more than $20 \mathrm{~m}$ for wavelengths greater than $400 \mathrm{~nm}$. [7] A typical $45 \mathrm{MeV}$ electron created in the detector produces a total of $\sim 1500$ photoelectrons, of which $\sim 280$ photoelectrons are in the Čerenkov cone. The phototube time and pulse height signals are used to reconstruct the track with an average r.m.s. position resolution of $\sim 30 \mathrm{~cm}$, an angular resolution of $\sim 12$ degrees, and an energy resolution of $\sim 7 \%$. The Čerenkov cone for relativistic particles and the time distribution of the light, which is broader for non-relativistic particles, give excellent particle identification. Surrounding the detector is a veto shield [8] which tags cosmic ray muons going through the detector. 


\section{DATA ANALYSIS}

The primary oscillation search in LSND is the search for $\bar{\nu}_{\mu} \rightarrow \bar{\nu}_{e}$ oscillations, where the $\bar{\nu}_{\mu}$ arise from $\mu^{+}$decay at rest in the beam stop and the $\bar{\nu}_{e}$ are identified through the reaction $\bar{\nu}_{e} p \rightarrow e^{+} n$. This reaction allows a two-fold signature of a positron with a $52 \mathrm{MeV}$ endpoint and a correlated $2.2 \mathrm{MeV} \gamma$ from neutron capture on a free proton. The positron/electron selection criteria (LSND is unable to determine charge) for this primary oscillation search is the following. First, in order to eliminate muon decay events, it is required that there be no event within $8 \mu$ s in the future or within $12 \mu$ s in the past. Second, the event particle identification parameter, $\chi_{p}$, is required to lie in the range $-1.5<\chi_{p}<0.5$, where the precise range values are determined by maximizing the acceptance divided by the square root of the beam-off background. Third, there must be less than 4 veto hits associated with the event and the time of the nearest veto hit must be more than 30 ns from the event time. Fourth, the positron energy is required to be in the range $20<E_{e}<60 \mathrm{MeV}$. Fifth, the event reconstructed position must be more than $35 \mathrm{~cm}$ from the nearest phototube surface. Finally, it is required that there be no more than one correlated $\gamma$ with $R_{\gamma}>10$ (see below) in order to reduce the background from cosmic-ray neutrons, which will typically knock-out additional neutrons.

The correlated $2.2 \mathrm{MeV} \gamma$ selection criteria makes use of the likelihood ratio, $R_{\gamma}$, which is defined to be the likelihood that the $\gamma$ is correlated divided by the likelihood that the $\gamma$ is accidental. $R_{\gamma}$ depends on three quantities: the number of hit phototubes associated with the $\gamma$ (the multiplicity is proportional to the $\gamma$ energy), the distance between the reconstructed $\gamma$ and positron positions, and the time between the $\gamma$ and positron. As checks of the likelihood distributions, Figs. 1 and 2 show the $R_{\gamma}$ distributions for $\nu_{e} C \rightarrow e^{-} N_{g s}$ exclusive events, where the $N_{g s}$ beta decays, and $\nu_{\mu} C \rightarrow \mu^{-} X$ and $\bar{\nu}_{\mu} C \rightarrow \mu^{+} X$ and $\bar{\nu}_{\mu} p \rightarrow \mu^{+} p$ inclusive events. By definition, the former reaction has no recoil neutron, so that its $R_{\gamma}$ distribution should be consistent with a purely accidental $\gamma$ distribution; indeed, a fit to the $R_{\gamma}$ distribution finds that the fraction of events with a correlated $\gamma$, $f_{c}$, is $f_{c}=-0.004 \pm 0.007\left(\chi^{2}=4.6 / 9 \mathrm{DOF}\right)$. For the latter reactions, correlated $\gamma$ are expected for $\sim 14 \%$ of the events. [9] A fit to the $R_{\gamma}$ distribution gives $f_{c}=0.129 \pm 0.013\left(\chi^{2}=8.2 / 9 \mathrm{DOF}\right)$, in agreement with expectations. Note that with the new reconstruction, the correlated $\gamma$ efficiency has increased while the accidental $\gamma$ efficiency has decreased. For $R_{\gamma}>10$, the correlated and accidental efficiencies are 0.3929 and 0.0026 , respectively. With the old reconstruction the correlated and accidental efficiencies were 0.230 and 0.006 , respectively.

The secondary oscillation search in LSND is the search for $\nu_{\mu} \rightarrow \nu_{e}$ oscillations, where the $\nu_{\mu}$ arise from $\pi^{+}$decay in flight in the beam stop and the $\nu_{e}$ are identified through the reaction $\nu_{e} C \rightarrow e^{-} X$. The electron selection criteria for this primary oscillation search is almost the same as for the primary search, except that the electron energy is required to be in the range $60<E_{e}<200 \mathrm{MeV}$ and there must be no associated $2.2 \mathrm{MeV} \gamma$. 


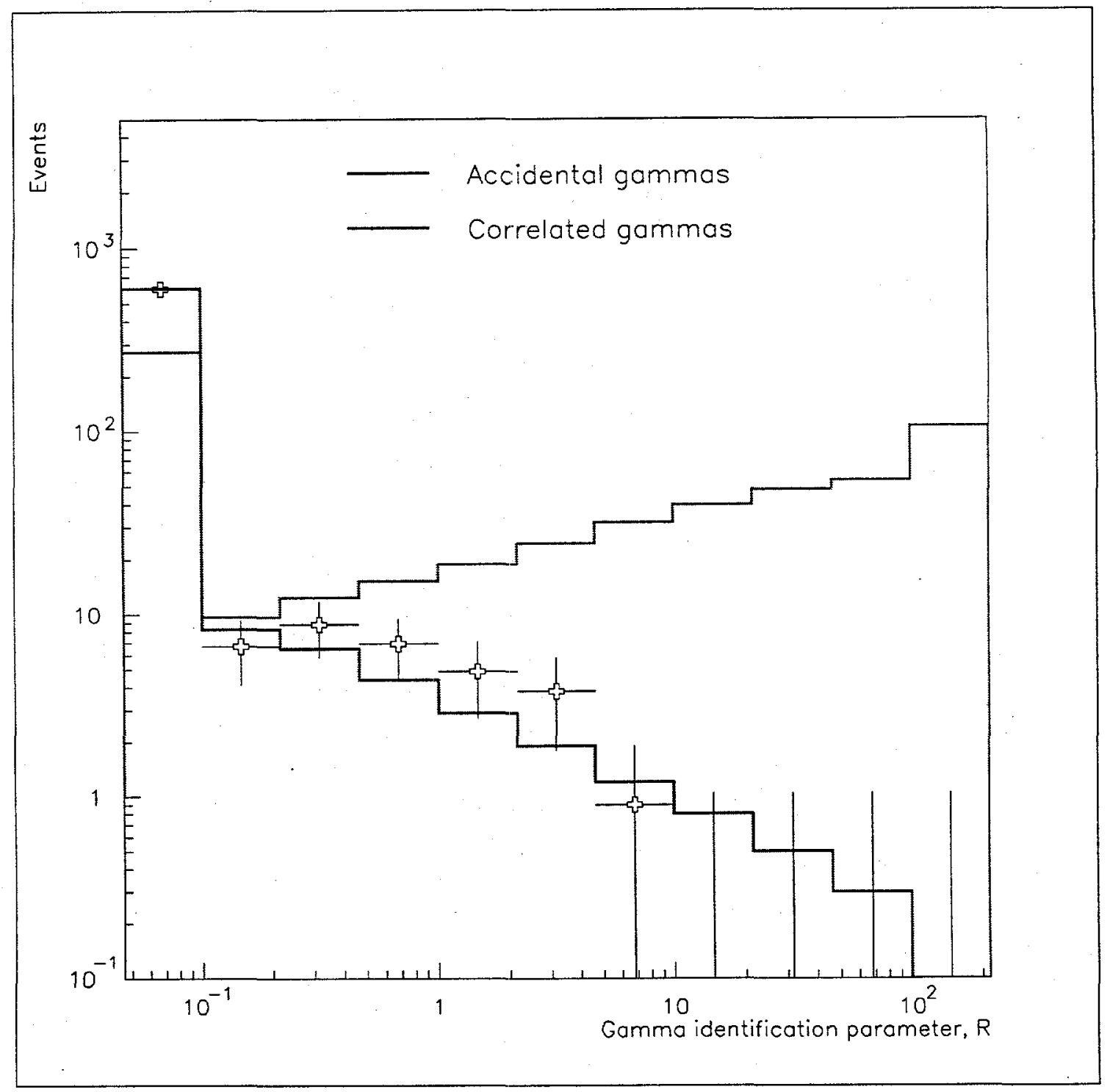

FIGURE 1. The $R_{\gamma}$ distribution for $\nu_{e} C \rightarrow e^{-} N_{g s}$ exclusive events, where the $N_{g s}$ beta decays. 


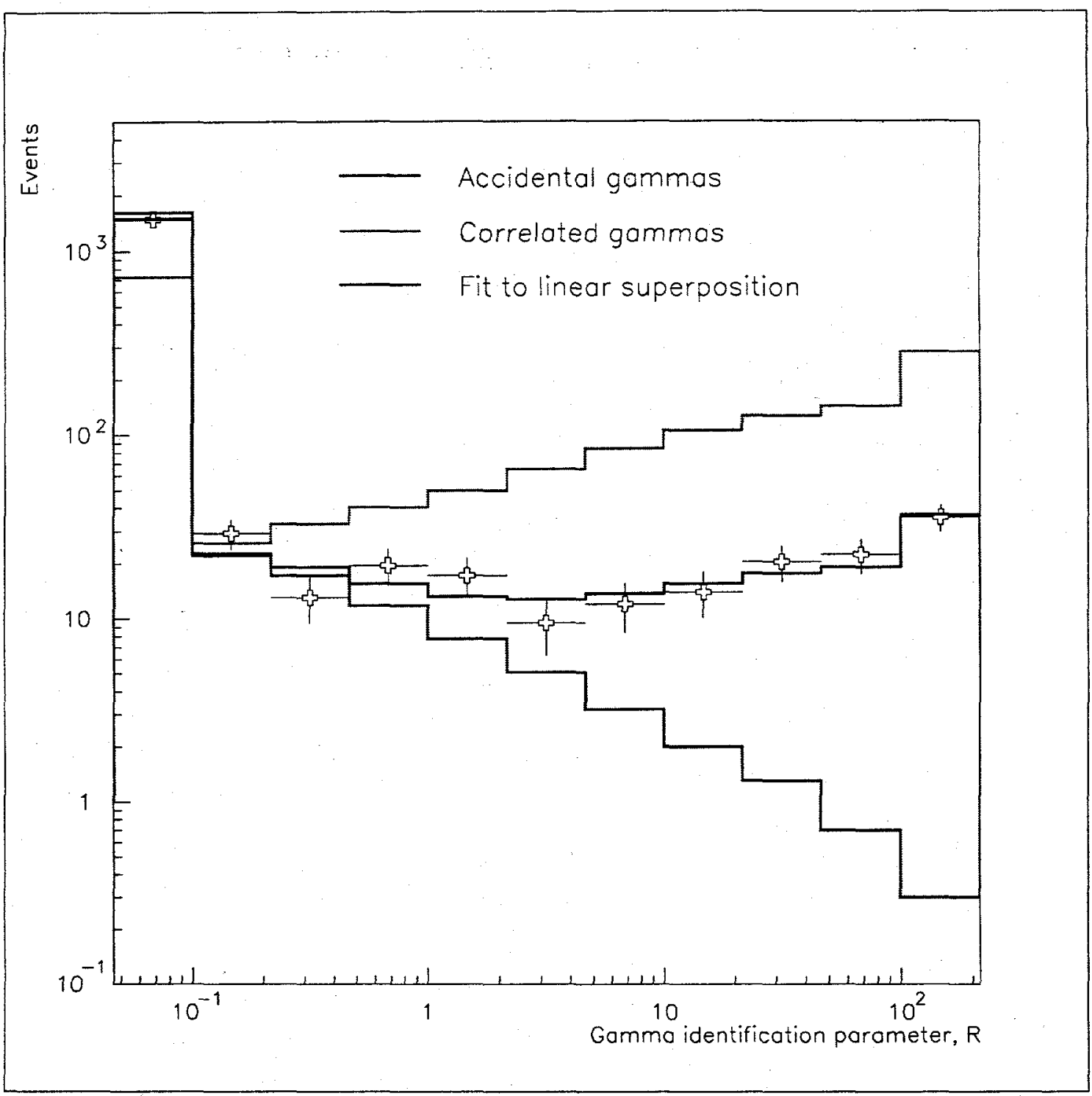

FIGURE 2. The $R_{\gamma}$ distribution for $\nu_{\mu} C \rightarrow \mu^{-} X$ and $\bar{\nu}_{\mu} C \rightarrow \mu^{+} X$ and $\vec{\nu}_{\mu} p \rightarrow \mu^{+} p$ inclusive events. 
TABLE 1. Numbers of beam-on events that satisfy the selection criteria for the primary $\bar{\nu}_{\mu} \rightarrow \bar{\nu}_{e}$ oscillation search with $R_{\gamma}>1, R_{\gamma}>10$, and $R_{\gamma}>100$. Also shown are the correlated $\gamma$ efficiencies, the beam-off background, the estimated neutrino background, and the excess of events that is consistent with neutrino oscillations.

\begin{tabular}{|c|c|c|c|c|}
\hline Selection \& Efficiency & Beam-On Events & Beam-Off Background & $\nu$ Background & Event Excess \\
\hline$R_{\gamma}>1(51.15 \%)$ & 195 & $98.1 \pm 2.4$ & 37.7 & $59.2 \pm 14.2$ \\
$R_{\gamma}>10(39.29 \%)$ & 83 & $33.7 \pm 1.4$ & 16.6 & $32.7 \pm 9.2$ \\
$R_{\gamma}>100(16.86 \%)$ & 25 & $7.9 \pm 0.7$ & 5.4 & $11.7 \pm 5.0$ \\
\hline
\end{tabular}

\section{NEUTRINO OSCILLATION RESULTS}

Table 1 gives the statistics for events that satisfy the selection criteria for the primary $\bar{\nu}_{\mu} \rightarrow \bar{\nu}_{e}$ oscillation search. An excess of events is observed over what is expected from beam-off and neutrino background that is consistent with neutrino oscillations. A fit to the $R_{\gamma}$ distribution, as shown in Fig. 3, gives $f_{c}=0.0578 \pm$ $0.0108\left(\chi^{2}=9.2 / 9 \mathrm{DOF}\right)$, which leads to a beam on-off excess of $113.3 \pm 21.2$ events with a correlated neutron. Subtracting the neutrino background from $\mu^{-}$ decay at rest followed by $\bar{\nu}_{e} p \rightarrow e^{+} n$ scattering (21.6 events) and $\pi^{-}$decay in flight followed by $\bar{\nu}_{\mu} p \rightarrow \mu^{+} n$ scattering (8.4 events) [10] leads to a total excess of $83.3 \pm 21.2$ events or an oscillation. probability of $(0.25 \pm 0.06 \pm 0.04) \%$. (Note that with the old reconstruction the oscillation probability was determined to be $(0.33 \pm 0.09 \pm 0.05) \%$.)

A fairly clean sample of oscillation candidate events can be obtained by requiring $R_{\gamma}>10$, where as shown in Table 1 , the beam on-off excess is $49.3 \pm 9.2$ events while the estimated neutrino background is only 16.6 events. Fig. 4 displays the energy distribution of events with $R_{\gamma}>10$. The shaded region shows the estimated neutrino background while the curves show the expected distributions from a combination of neutrino background plus neutrino oscillations at high or low $\Delta m^{2}$. The data agree well with the oscillation hypothesis. Fig. 5 shows the spatial distribution for events with $R_{\gamma}>10$, where $\mathrm{z}$ is along the axis of the tank (and approximately along the beam direction), $\mathrm{y}$ is vertical, and $\mathrm{x}$ is transverse. The data agree well with the distributions from $\nu_{e} C \rightarrow e^{-} N_{g s}$ scattering (solid histogram), where the reaction is identified by the $N_{g s}$ beta decay.

A test of the oscillation hypothesis is to check whether there is an excess of events with $>1$ correlated $\gamma$. If the excess of events is indeed due to the reaction $\bar{\nu}_{e} p \rightarrow e^{+} n$, then there should be no excess with $>1$ correlated $\gamma$ because the recoil $n$ is too low in energy $(<5 \mathrm{MeV})$ to knock out additional neutrons. If, on the other hand, the excess involves higher energy neutrons $(>20 \mathrm{MeV})$, then one would expect a comparable excess with $>1$ correlated $\gamma$. However, as shown in Table 2, the excess of events with $>1$ correlated $\gamma$ is approximately zero, as expected for the reaction $\bar{\nu}_{e} p \rightarrow e^{+} n$. 


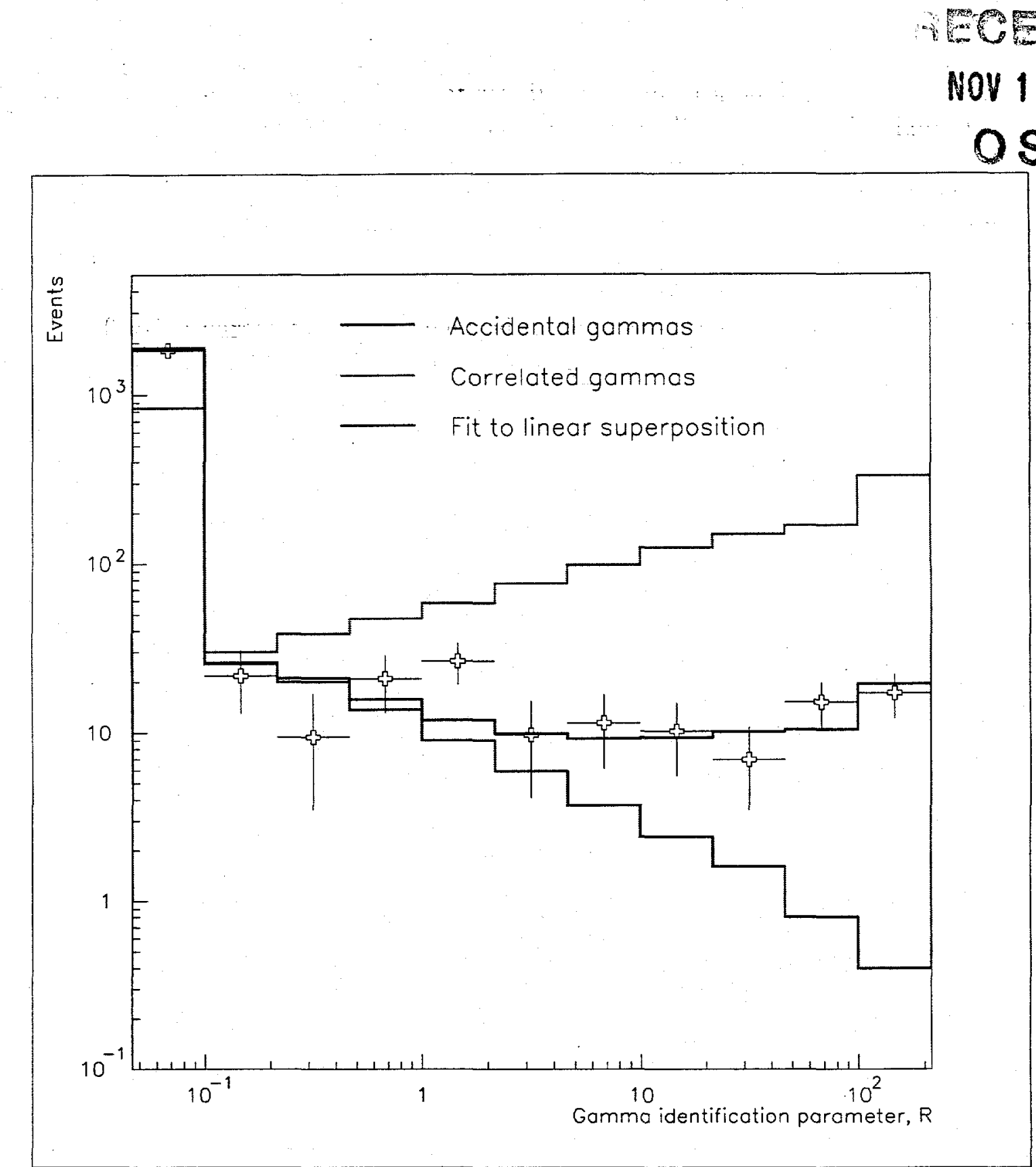

FIGURE 3. The $R_{\gamma}$ distribution for events that satisfy the selection criteria for the primary $\bar{\nu}_{\mu} \rightarrow \bar{\nu}_{e}$ oscillation search. 


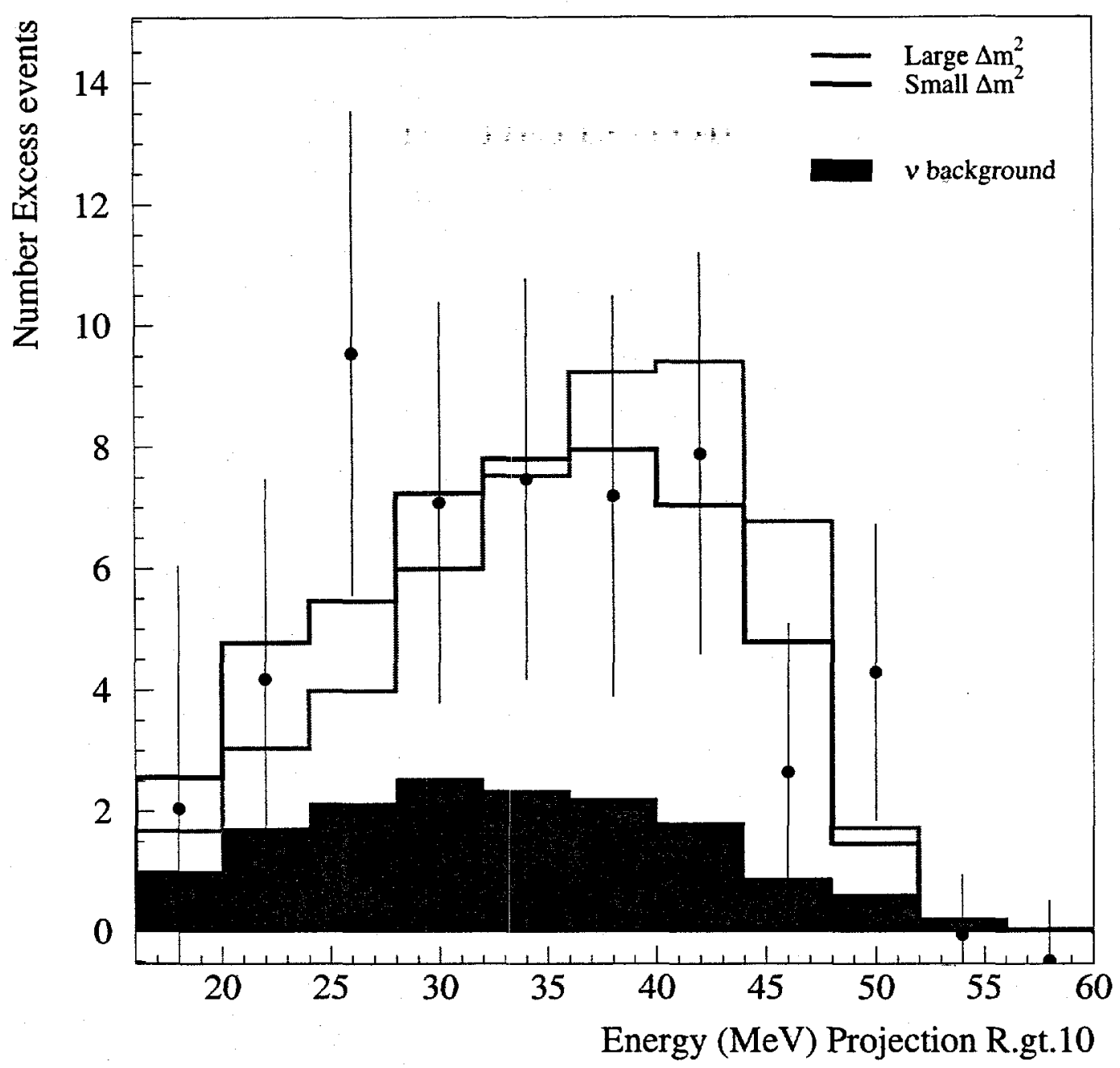

FIGURE 4. The energy distribution of events with $R_{\gamma}>10$. The shaded region shows the estimated neutrino background while the curves show the expected distributions from a combination of neutrino background plus neutrino oscillations at high or low $\Delta m^{2}$. 


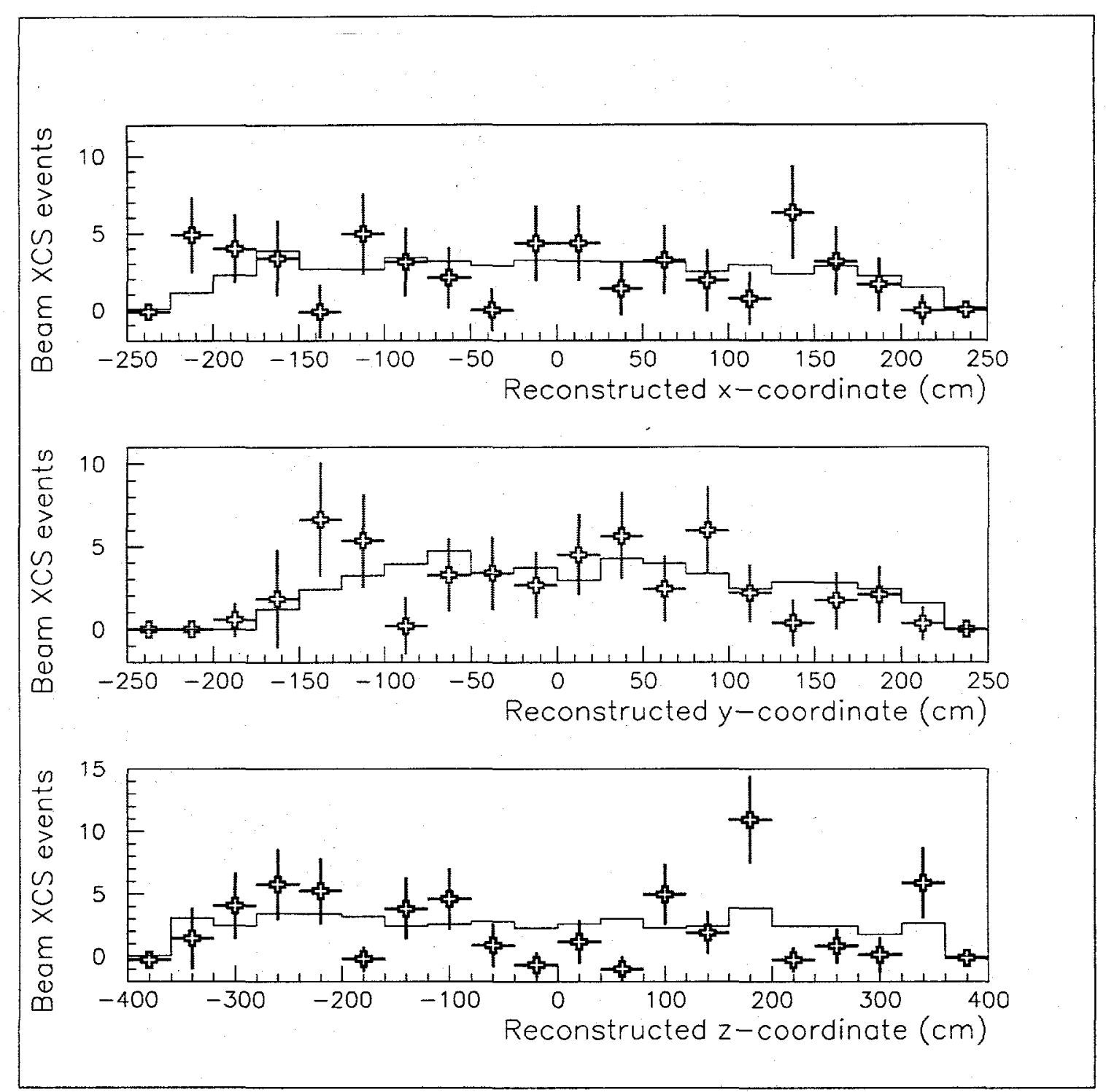

FIGURE 5. The spatial distributions for events with $R_{\gamma}>10$. The data agree well with the distributions from $\nu_{e} C \rightarrow e^{-} N_{g s}$ scattering (solid histogram), where the reaction is identified by the $N_{g s}$ beta decay. 
TABLE 2. Number of beam on-off excess events that satisfy the selection criteria for the primary $\bar{\nu}_{\mu} \rightarrow \bar{\nu}_{e}$ oscillation search with one $R_{\gamma}>10$ associated $\gamma$ and with $>1 R_{\gamma}>10$ associated $\gamma$. The excess of events with $>1$ correlated $\gamma$ is approximately zero, which is what is expected for the reaction $\bar{\nu}_{e} p \rightarrow e^{+} n$.

\begin{tabular}{|c|c|c|}
\hline Energy Selection & 1 Associated $\gamma$ & $>1$ Associated $\gamma$ \\
\hline $20<E_{e}<60 \mathrm{MeV}$ & $49.2 \pm 9.1$ & $-2.8 \pm 1.7$ \\
$36<E_{e}<60 \mathrm{MeV}$ & $20.8 \pm 5.8$ & $-2.8 \pm 1.0$ \\
\hline
\end{tabular}

A $\Delta m^{2}$ vs. $\sin ^{2} 2 \theta$ oscillation parameter fit for the entire data sample, $20<$ $E_{e}<200 \mathrm{MeV}$, is shown in Fig. 6. The fit includes both $\bar{\nu}_{\mu} \rightarrow \bar{\nu}_{e}$ and $\nu_{\mu} \rightarrow \nu_{e}$ oscillations, as well as all known neutrino backgrounds. The red and blue regions correspond to $90 \%$ and $99 \%$ CL allowed regions, while the curves are $90 \%$ CL limits from the Bugey reactor experiment, [11] the CCFR experiment at Fermilab, [12] the NOMAD experiment at CERN, [13] and the KARMEN experiment at ISIS. [14] The most favored allowed region is the band from 0.2 to $2.0 \mathrm{eV}^{2}$, although a region around $7 \mathrm{eV}^{2}$ is also possible. Note that a fit to the $60<E_{e}<200 \mathrm{MeV}$ data sample, involving secondary $\nu_{\mu} \rightarrow \nu_{e}$ oscillations only, results in a total excess of $8.3 \pm 5.5$ oscillation events or an oscillation probability of $(0.09 \pm 0.06 \pm 0.04) \%$, which is consistent with what is expected at low $\Delta m^{2}\left(\Delta m^{2}<2 \mathrm{eV}^{2}\right)$.

\section{CONCLUSIONS}

The LSND experiment provides evidence for neutrino oscillations from both the primary $\bar{\nu}_{\mu} \rightarrow \bar{\nu}_{e}$ oscillation search and the secondary $\nu_{\mu} \rightarrow \nu_{e}$ oscillation search. At present, this remains the only evidence for appearance neutrino oscillations and implies that at least one neutrino has a mass greater than $0.4 \mathrm{eV} / \mathrm{c}^{2}$ and that neutrinos comprise more than $1 \%$ of the mass of the universe. The MiniBooNE experiment at Fermilab, [15] which is presently under construction, will provide a definitive test of the LSND results, and if the neutrino oscillation results are confirmed, will make a precision measurement of the oscillation parameters.

\section{REFERENCES}

1. The LSND Collaboration presently consists of the following people and institutions: E. D. Church, I. Stancu, W. Strossman, G.J. VanDalen (Univ. of California, Riverside); W. Vernon (Univ. of California, San Diego); D.O. Caldwell, S. Yellin (Univ. of California, Santa Barbara); D. Smith (Embry-Riddle Aeronautical Univ.); R.L. Burman, J.B. Donahue, G.T. Garvey, W.C. Louis, G.B. Mills, V. Sandberg, R. Tayloe, D.H. White (Los Alamos National Laboratory);R. Imlay, H.J. Kim, A. Malik, 


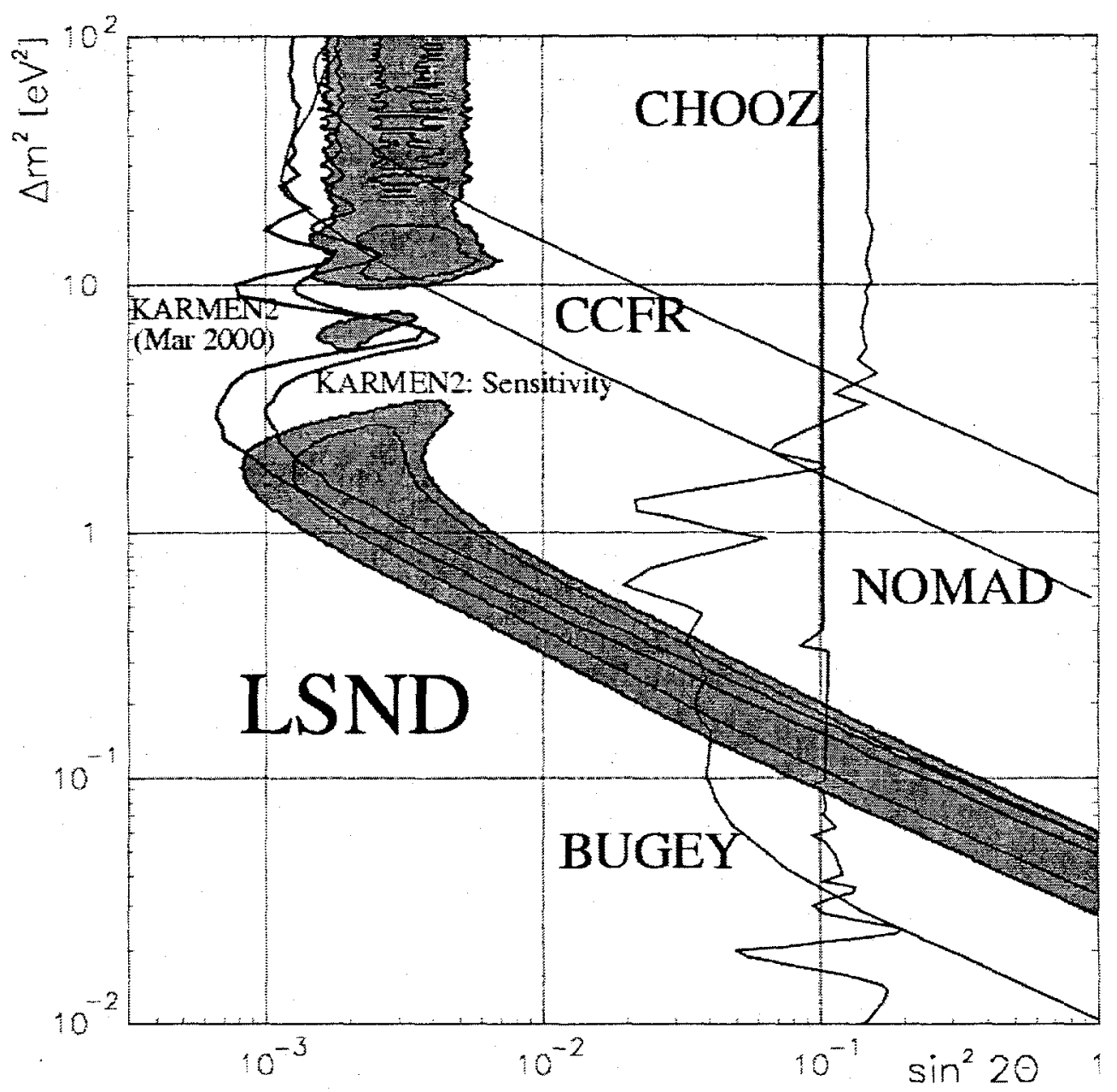

FIGURE 6. A $\Delta m^{2}$ vs. $\sin ^{2} 2 \theta$ oscillation parameter fit for the entire data sample, $20<E_{e}<200 \mathrm{MeV}$. The fit includes primary $\bar{\nu}_{\mu} \rightarrow \bar{\nu}_{e}$ oscillations and secondary $\nu_{\mu} \rightarrow \nu_{e}$ oscillations, as well as all known neutrino backgrounds. The red and blue regions correspond to $90 \%$ and $99 \%$ CL allowed regions, while the red curves are $90 \%$ CL limits from the Bugey reactor experiment, the CCFR experiment at Fermilab, the NOMAD experiment at CERN, and the KARMEN experiment at ISIS. 
W. Metcalf, M.K. Sung, N. Wadia (Louisiana State Univ.): K. Johnston (Louisiana Tech Univ.); A. Fazely, R.M. Gunasingha (Southern Univ); L.B. Auerbach, R. Majkic (Temple Univ.).

2. C. Athanassopoulos et al., Phys. Rev. Lett. 75, 2650 (1995).

3. C. Athanassopoulos et al., Phys. Rev. C 54, 2685 (1996); C. Athanassopoulos et al., Phys. Rev. Lett. 77, 3082 (1996).

4. C. Athanassopoulos et al., Phys. Rev. Lett. 81, 1774 (1998); C. Athanassopoulos et al., Phys. Rev. C 58, 2489 (1998).

5. C. Athanassopoulos et al., Nucl. Instrum. Methods A 388, 149 (1997).

6. R.L. Burman, M.E. Potter, and E.S. Smith, Nucl. Instrum. Methods A 291, 621 (1990); R.L. Burman, A.C. Dodd, and P. Plischke, Nucl. Instrum. Methods in Phys. Res. A 368, 416 (1996).

7. R.A. Reeder et al., Nucl. Instrum. Methods A 334, 353 (1993).

8. J.J. Napolitano et al., Nucl. Instrum. Methods A 274, 152 (1989).

9. E. Kolbe, K. Langanke, F.-K. Thielmann, and P. Vogel, Phys. Rev. C 52, 3437 (1995).

10. This background estimate also includes contributions from $\bar{\nu}_{\mu} C \rightarrow \mu^{+} n X$ and $\nu_{\mu} C \rightarrow$ $\mu^{-} n X$.

11. B. Achkar et al., Nucl. Phys. B434, 503 (1995).

12. A. Romosan et al., Phys. Rev. Lett. 78, 2912 (1997).

13. D. Autiero, talk presented at the 1998 International Conference on High Energy Physics in Vancouver, Canada.

14. K. Eitel, talk presented at the 2000 International Neutrino Conference in Sudbury, Canada.

15. E. Church et al., "A proposal for an experiment to measure $\nu_{\mu} \rightarrow \nu_{e}$ oscillations and $\nu_{\mu}$ disappearance at the Fermilab Booster: BooNE", LA-UR-98-352, Fermilab experiment 898 . 